Article

\title{
Strong Convergence Theorems for Variational Inequalities and Common Fixed-Point Problems Using Relaxed Mann Implicit Iteration Methods
}

\author{
Lu-Chuan Ceng ${ }^{1}$ and Meijuan Shang ${ }^{2, *}$ \\ 1 Department of Mathematics, Shanghai Normal University, Shanghai 201416, China; zenglc@hotmail.com \\ 2 College of Science, Shijiazhuang University, Shijiazhuang 266100, China \\ * Correspondence: meijuanshang@sjzc.edu.cn
}

Received: 10 March 2019; Accepted: 7 May 2019; Published: 11 May 2019

\begin{abstract}
Mann-like iteration methods are significant to deal with convex feasibility problems in Banach spaces. We focus on a relaxed Mann implicit iteration method to solve a general system of accretive variational inequalities with an asymptotically nonexpansive mapping in the intermediate sense and a countable family of uniformly Lipschitzian pseudocontractive mappings. More convergence theorems are proved under some suitable weak condition in both 2-uniformly smooth and uniformly convex Banach spaces.
\end{abstract}

Keywords: relaxed Mann implicit iteration; general variational inequalities; countable many uniformly Lipschitzian pseudocontractions; asymptotically nonexpansive mapping in the intermediate sense; banach space

MSC: 47H09; 47J20; 49M05

\section{Introduction}

Throughout this article, $E$ will be supposed to be a real Banach space and $E^{*}$ stands for its topological dual. Recall the normalized duality mapping $J: E \rightarrow 2^{E^{*}}$ as follows

$$
J x:=\left\{\varphi \in E^{*}:\langle x, \varphi\rangle=\|x\|^{2},\|\varphi\|=\|x\|\right\} \forall x \in E .
$$

The duality $J$ will be reduced to the identical operator in setting of Hilbert space. Set $U S_{E}=$ $\{x \in E:\|x\|=1\}$. E is said to be a smooth space if $\lim _{t \rightarrow 0}\left(\mid x / t+y \|-\frac{\|x\|}{t}\right)$ exists for all $x, y \in U S_{E}$. If $E$ is smooth, then normalized duality is a single-valued operator. For the rest of this paper, we shall invoke $j$ the normalized duality with the single-valued. $E$ is said to be a uniformly smooth Banach space if the above limit is uniformly achieved for all $x, y \in U S_{E}$.

Let $C$ be a closed nonempty and convex set in $E$. Invoke that the operator $f$ is said to be $\delta$-Lipschitzian continuous in the set $C$ if

$$
\|f(x)-f(y)\| \leq \delta\|x-y\|, \quad \forall x, y \in C,
$$

where $\delta$ is a real number in $[0,+\infty)$. The existence of solutions of many abstract problems is equivalent to the existence of a fixed point of a Lipschitzian continuous mapping. Therefore, existence results of Lipschitzian continuous mappings are significant in mathematical sciences. If $f$ is $\delta$-Lipschitzian continuous with $\delta<1$, then $f$ is called a $\delta$-contraction. Each contractive operator equation $f: C \rightarrow C$ has a unique solution in $C$ according to the famous Banach contractive mapping principal. Invoke that the mapping $f$ is said to be a nonexpansive mapping when $\delta=1$. For the rest of the paper, we use 
Fix $(f)$ to denote the set of fixed points of $f$. Invoke that the mapping $T$ is said to be asymptotically nonexpansive [1] when

$$
\left\|T^{n} x-T^{n} y\right\| \leq\left(\theta_{n}+1\right)\|x-y\|, \quad \forall x, y \in C, n \geq 0 .
$$

Here $\left\{\theta_{n}\right\}$ is in the interval $[0,+\infty)$ with $\lim _{n \rightarrow \infty} \theta_{n}=0$. If the operator $T$ is continuous and satisfies

$$
\limsup _{n \rightarrow \infty} \sup _{x, y \in C}\left(\left\|T^{n} x-T^{n} y\right\|-\|x-y\|\right) \leq 0,
$$

then the operator $T$ is called an asymptotically nonexpansive operator, which is in the intermediate sense; see [2]. It is easy to conclude that the family of asymptotically nonexpansive mappings, which is in the intermediate sense, as special cases, includes the one of contractive operators, the one of nonexpansive operators and the one of asymptotically nonexpansive operators. For the rest this paper, set

$$
c_{n}:=\max \left\{\sup _{x, y \in C}\left(\left\|T^{n} x-T^{n} y\right\|-\|x-y\|\right), 0\right\} .
$$

Then $c_{n} \geq 0 \forall n \geq 0, c_{n} \rightarrow 0(n \rightarrow \infty)$, and (1) become the inequality

$$
\left\|T^{n} x-T^{n} y\right\| \leq\|x-y\|+c_{n}, \quad \forall x, y \in C, \forall n \geq 0 .
$$

Solutions of nonlinear equations containing nonexpansive operators, asymptotically nonexpansive operators, and asymptotically nonexpansive operators in the intermediate sense recently attracted much attention from many authors; see [3-14] and the references therein.

Invoke that an operator $T$ with range $R(T)$ and domain $D(T)$ is called a pseudocontractive operator if

$$
\|x-y\| \leq\|x-y+r((I-T) x-(I-T) y)\|, \quad \forall x, y \in D(T), r>0 .
$$

From the result of Kato [15], we know that the definition of pseudocontractive operators is equivalent to: for each $x, y \in D(T),\langle T x-T y, j(x-y)\rangle \leq\|x-y\|^{2}$. Here $j(x-y) \in J(x-y)$ is the single-valued normalized duality. One can clearly see the situation the family of pseudocontractive operators is an important and significant generation of nonexpansive operators. Moreover, methodology shed on pseudocontractive operator is from their counterpart: accretivity (monotonicity in Hilbert spaces); see [16-22] and the references therein.

Let the Banach space $E$ be smooth. Let $B_{1}, B_{2}: C \rightarrow E$ be two mappings. The general system of variational inequalities (GSVI) is to find $\left(x^{*}, y^{*}\right) \in C \times C$ such that

$$
\begin{cases}\left\langle x^{*}-y^{*}+\mu_{1} B_{1} y^{*}, j\left(x-x^{*}\right)\right\rangle \geq 0, & \forall x \in C, \\ \left\langle y^{*}-x^{*}+\mu_{2} B_{2} x^{*}, j\left(x-y^{*}\right)\right\rangle \geq 0, & \forall x \in C,\end{cases}
$$

where $\mu_{1}$ and $\mu_{2}$ are two positive real constants. When $E$ become $H$ the real Hilbert space, the GSVI (3) becomes the GSVI considered in [23], which includes as special cases the problems arising, especially from linear or nonlinear complementary problems, and quadratic convex programming. It has no doubt the system of variational inequalities has played a crucial role on both theoretical and applied sciences. The literature on the variational inequalities is vast and some efficient methods have received great development. People extend them in various ways and extended them to develop new iterative algorithms for solving other relevant problems; see, e.g., [23-26]. It is worth emphasizing that an important method of solving the variational inequality is to transform it into a fixed-point problem. In 2017, Cai, Shehu and Iyiola [24] proposed and analyzed a generalized viscosity numerical algorithm for approximating solutions of the GSVI (3) in uniform spaces.

In the present paper, we introduce a relaxed Mann-like iteration method for the approximation of solutions of the GSVI (3) in both 2-uniformly smooth and uniformly convex Banach setting. The 
relaxed Mann implicit iteration method presented in this paper is from both the Mann iteration and the Korpelevich's extragradient iteration.

\section{Preliminaries}

Throughout this present paper we get $x_{n} \rightarrow x$ (resp., $x_{n} \rightarrow x$ ) to indicate that the sequence $\left\{x_{n}\right\}$ converges weakly (resp., strongly) to $x$. We invoke $C$ to be a convex nonempty closed set in $E$. Invoke that $j(x-y)$ stands for the single-valued normalized duality for any $x, y \in E$. A mapping $A: C \rightarrow E$ is said to be

(a) accretive if $\langle A x-A y, j(x-y)\rangle \geq 0, \forall x, y \in C$.

(b) $\alpha$-strongly accretive if $\langle A x-A y, j(x-y)\rangle \geq \alpha\|x-y\|^{2}$ for some $\alpha \in(0,1), \forall x, y \in C$.

(c) $\beta$-inverse-strongly accretive if $\langle A x-A y, j(x-y)\rangle \geq \beta\|A x-A y\|^{2}$ for some $\beta>0, \forall x, y \in C$.

(d) $\lambda$-strictly pseudocontractive if $\|x-y\|^{2}-\lambda\|(I-A) x-(I-A) y\|^{2} \geq\langle A x-A y, j(x-y)\rangle$ for some $\lambda \in(0,1), \forall x, y \in C$.

Definition 1. Fix a convex and closed set $C$ in a space $E$ and $\left\{S_{n}\right\}_{n=0}^{\infty}: C \rightarrow C$ stands for a self-operator sequence of continuous pseudocontractive. We say $\left\{S_{n}\right\}_{n=0}^{\infty}: C \rightarrow C$ is a family of $\ell$-uniformly Lipschitzian pseudocontractive operators when $S_{n}$ is $\ell$-Lipschitz continuous, where $\ell>0$ is a real constant.

For a linear operator $A$, we say it is strongly positive when

$$
\frac{\langle A x, j(x)\rangle}{\bar{\gamma}} \geq\|x\|^{2},\|b A-a I\|=\sup _{\|x\| \leq 1}|\langle(b A-a I) x, j(x)\rangle|, b \in[-1,1], a \in[0,1],
$$

where $\bar{\gamma}>0$ and $j(\cdot)$ is the single-valued duality mapping.

We say a Banach space $E$ is strictly convex if $y \neq x \Rightarrow\|y+x\|<2$ for any $x, y \in U S_{E}$. We say it is uniformly convex if there exists $\delta>0$ such that for any $x, y \in U S_{E},\|y-x\| \geq \epsilon \Rightarrow\|y+x\| \leq 2-2 \delta$, for each $\epsilon \in(0,2]$. one knows that a uniform space is the reflexive. Also, in the setting of reflexive spaces, $E$ is strictly convex if and only if $E^{*}$ is smooth as well as $E$ is smooth if and only if $E^{*}$ is strictly convex. A Banach space $E$ is called to have a Gateaux differentiable norm if the limit $\lim _{t \rightarrow \infty} t\left(\left\|\frac{1}{t} y+x\right\|-\|x\|\right)$ exists for each $x, y \in U S_{E}$ and in this case we call $E$ is smooth; $E$ is called to have a uniformly Gateaux differentiable norm if the above limit is achieved uniformly for any $x \in U$. Moreover, it is called to have a uniformly Fréchet differentiable norm if the above limit is attained uniformly for $x, y \in U S_{E}$ and in this case we call $E$ uniformly smooth. The norm of $E$ is said to be the Fréchet differentiable if for each $x \in U$, the above limit is uniformly achieved for $y \in U$. The modulus of smoothness of $E$ is defined by

$$
\varrho(\tau)=\sup \left\{\frac{\|x+y\|-2+\|x-y\|}{2}: x, y \in E,\|x\|=1, \tau=\|y\|\right\},
$$

where the function $\varrho$ maps $[0, \infty)[0, \infty)$. Invoke that $E$ is uniformly smooth if $\frac{\varrho(\tau)}{\tau} \rightarrow 0$ as $\tau \rightarrow 0$. Let $q$ $(q>1$ and $q \leq 2)$ be a fixed real number. $E$ is said to be $q$-uniformly smooth if $c \tau^{q} \geq \varrho(\tau), \forall \tau>0$, $c>0$. One has $\|y+x\|^{q} \leq 2\left(\|x\|^{q}+\|\kappa y\|^{q}\right)-\|x-y\|^{q}$, where $\kappa>0$ in the setting of $q$-uniformly smooth spaces. The best number $\kappa$ in this inequality is named to be the $q$-uniformly smooth number of E; see [26].

For $q>1$, one can define the generalized duality mapping $J_{q}$ by

$$
J_{q}(x)=\left\{\varphi \in E^{*}:\langle x, \varphi\rangle=\|x\|^{q},\|\varphi\|=\|x\|^{q-1}\right\}, \quad \forall x \in E .
$$

It is known that $\forall p \in E, J(p)=\frac{J_{q}(p)}{\|p\|^{q-2}}$. If the setting is Hilbert, then $J$ become $I$, the identity. Recall that the following statements hold: 
(1) $J$ is norm-to-weak* continuous and single-valued in setting of smooth spaces;

(2) $J$ is norm-to-norm uniformly continuous on bounded sets and single-valued in setting of uniformly smooth spaces;

(3) 2-uniformly smooth spaces are: Hilbert spaces, the Sobolev spaces $W_{m}^{p}$, and $L^{p}$ spaces, where $p \geq 2$;

(4) $L^{p}$ with $p>1$, is uniformly convex and uniformly smooth.

Proposition 1 ([27]). Fix a convex nonempty closed set $C$ in a Banach space $E$. Let $S_{0}, S_{1}, \ldots: C \rightarrow C$ be a self-mapping sequence. Suppose that $\sum_{n=1}^{\infty} \sup \left\{\left\|\left(S_{n}-S_{n-1}\right) x\right\|: x \in C\right\}$ is a fixed real number. Then $\left\{S_{n} y\right\}$, where $y \in C$, converges in norm. If the mapping is defined by $S y=\lim _{n \rightarrow \infty} S_{n} y$ for all $y \in C$, then $\sup \left\{\left\|S x-S_{n} x\right\|: x \in C\right\} \rightarrow 0$ as $n \rightarrow \infty$.

Proposition 2 ([28]). Fix a convex nonempty closed set $C$ in a Banach space E. Every continuous and strong pseudocontraction self-mapping $T: C \rightarrow C$ has a fixed point. Furthermore, it has only a unique fixed point.

Fix a set $D$ in the set $C$ and let projective mapping $\Pi$ map $C$ into $D$. Then $\Pi$ is named as a sunny if $\Pi[t(x-\Pi(x))+\Pi(x)]=\Pi(x)$, whenever $t(x-\Pi(x))+\Pi(x) \in C$ for all $x \in C$ and for all $t \geq 0$. A projective mapping $\Pi$ of $C$ into itself is named a retraction once $\Pi \Pi x=\Pi x$. Set $D$ is called is called a sunny nonexpansive retract of set $C$ if there is a $C-D^{\prime}$ s sunny nonexpansive retraction.

In a setting of smooth Banach spaces, $J$ is called a weakly sequentially continuous mapping if $\left\{j\left(x_{n}\right)\right\}$ converges weakly* to $j(x)$ for each $\left\{x_{n}\right\} \subset E$, here, $x_{n} \rightarrow x$. From [29], any space with a weakly continuous duality mapping satisfies the Opial inequality. In addition, if a space with the Opial inequality and a uniformly Gáteaux differentiable norm, then it enjoys duality mappings are weakly continuous.

Proposition 3. Fix a convex nonempty and closed set $C$ in a smooth Banach space $E$, set $D$ as a nonempty subset of $C$. If $\Pi: C \rightarrow D$ be a retraction, one has (i) $\Pi$ is both nonexpansive and sunny; (ii) $\langle x-y, j(\Pi(x)-$ $\Pi(y))\rangle \geq\|\Pi(x)-\Pi(y)\|^{2}, \forall x, y \in C$; (iii) for any $x \in C, y \in D,\langle x-\Pi(x), j(y-\Pi(x))\rangle \leq 0$.

One knows that any sunny nonexpansive retraction coincides with the metric projection in setting of a Hilbert space.

Proposition 4. Fix a convex nonempty and closed set $C$ in a reflexive Banach space $E$. If $E$ has a uniformly Gáteaux differentiable norm and the set $D$ is a nonexpansive retract in $C$, it is a sunny nonexpansive retract of $C$.

Proposition 5 ([30]). In the setting of 2-uniformly smooth Banach space,

$$
\|x+y\|^{2}-2\langle y, j(x)\rangle \leq\|x\|^{2}+2\|\kappa y\|^{2},
$$

where $\kappa$ is the 2-uniformly smooth constant of $E$.

In particular, if the duality pairing $\langle\cdot, \cdot\rangle$ become the inner product, $j=I$ the identity mapping of $E$, and $\kappa=\sqrt{2} / 2$ in setting of Hilbert space.

For obtaining our theorem, we need to invoke some lemmas in the sequel. The following lemma is obvious.

Lemma 1. Let $j$ be the normalized single-valued duality mapping on a real Banach space E. For any given $x, y \in E$, it holds $\mid x\left\|^{2}+2\langle y, j(x+y)\rangle \geq\right\| x+y \|^{2}$.

Lemma 2 ([31]). Fix a convex nonempty and closed set $C$ in a smooth Banach space $E$. Let $\Pi_{C}: E \rightarrow C$ be a nonexpansive sunny retraction and $B: C \rightarrow E$ be an accretive mapping. Then $\operatorname{VI}(C, B)=\operatorname{Fix}\left(\Pi_{C}(I-\right.$ 
$\lambda B)) \forall \lambda>0$, where $\operatorname{VI}(C, B)$ denotes the set of solutions to the VI of approximating $x^{*} \in C$ such that $\left\langle B x^{*}, j\left(x-x^{*}\right)\right\rangle \geq 0 \forall x \in C$.

Using Propositions 3 and 5, respectively, we immediately obtain the lemmas.

Lemma 3. Fix a convex nonempty and closed set $C$ in a smooth Banach space E. Let $B_{1}, B_{2}: C \rightarrow E$ be two nonlinear mappings. Let $\Pi_{C}: E \rightarrow C$ be a nonexpansive sunny retraction. For given $x^{*}, y^{*} \in C,\left(x^{*}, y^{*}\right)$ solves the GSVI (3) iff $x^{*} \in \operatorname{GSVI}\left(C, B_{1}, B_{2}\right)$ where $\operatorname{GSVI}\left(C, B_{1}, B_{2}\right)$ stands for fixed-point set of $G:=$ $\Pi_{C}\left(I-\mu_{1} B_{1}\right) \Pi_{C}\left(I-\mu_{2} B_{2}\right)$ and $y^{*}=\Pi_{C}\left(I-\mu_{2} B_{2}\right) x^{*}$.

Lemma 4. Fix a convex nonempty and closed set $C$ in a 2-uniformly smooth Banach space E. Let the accretive mapping $A: C \rightarrow E$ be $\alpha$-inverse-strongly. Then, for any given $\lambda \geq 0,\|x-y\|^{2}+2 \lambda\left(\kappa^{2} \lambda-\alpha\right) \| A y-$ $A x\left\|^{2} \geq\right\|(I-\lambda A) y-(I-\lambda A) x \|^{2}$. In particular, if $0 \leq \lambda \leq \frac{\alpha}{\kappa^{2}}$, then $I-\lambda A$ is nonexpansive.

Using Lemma 4, we immediately obtain the following lemma.

Lemma 5. Fix a convex nonempty and closed set $C$ in a 2-uniformly smooth Banach space $E$. Let $\Pi_{C}: E \rightarrow C$ be a nonexpansive sunny retraction. Let the accretive mappings $B_{1}, B_{2}: C \rightarrow E$ be $\alpha$-inverse-strongly and $\beta$-inverse-strongly accretive. Let the mapping $G: C \rightarrow C$ be defined as $G:=\Pi_{C}\left(\Pi_{C}\left(I-\mu_{2} B_{2}\right)-\right.$ $\left.\mu_{1} B_{1} \Pi_{C}\left(I-\mu_{2} B_{2}\right)\right)$. If $\alpha \geq \kappa^{2} \mu_{1} \geq 0$ and $\beta \geq \kappa^{2} \mu_{2} \geq 0$, then $G: C \rightarrow C$ is a nonexpansive operator.

Proposition 6 ([32]). Fix a convex nonempty and closed set $C$ in a uniformly convex Banach space $E$ and set $T$ an asymptotically nonexpansive self-mapping in the intermediate sense on $C$. Given any bounded subset $\mathcal{K} \subset C$. For every $\varepsilon>0$ and every $n \geq 2$ it is an integer $N_{\varepsilon} \geq 1$ and $\delta_{\varepsilon}>0$, where both $N_{\varepsilon}$ and $\delta_{\varepsilon}$ are independent of $n$, such that if $z_{1}, z_{2}, \ldots, z_{n} \in \mathcal{K}, k \geq N_{\varepsilon}$, and if $\left\|z_{i}-z_{j}\right\|-\left\|T^{k} z_{i}-T^{k} z_{j}\right\| \leq \delta_{\varepsilon}$ for $1 \leq i, j \leq n$, then

$$
\left\|T^{k}\left(\sum_{i=1}^{n} \lambda_{i} z_{i}\right)-\sum_{i=1}^{n} \lambda_{i} T^{k} z_{i}\right\|<\varepsilon
$$

for all $\lambda:=\left(\lambda_{1}, \lambda_{2}, \ldots, \lambda_{n}\right)$ such that $\lambda_{i} \geq 0$ for $i=1,2, \ldots, n$ and $\sum_{i=1}^{n} \lambda_{i}=1$.

One can make use of Proposition 6 to prove the lemmas below. We omit the proof.

Lemma 6. Fix a convex nonempty and closed set $C$ in a uniformly convex Banach space and set $T$ a uniformly continuous self-mapping on $C$, which is asymptotically nonexpansive in the intermediate sense. If $\left\{x_{m}\right\}_{m=0}^{\infty}$ converges in weak topology to $x$ and if $\left\|x_{m}-T x_{m}\right\| \rightarrow 0$ as $m \rightarrow \infty$, then $T x=x$.

Lemma 7. Fix a convex nonempty and closed set $C$ in a smooth Banach space E. If the accretive operator $A: C \rightarrow E$ is weakly continuous along segments (i.e., $A(t y+x) \rightarrow A x$ as $t \rightarrow 0)$, then the VI of approximating $x^{*} \in C$ such that $\left\langle A x^{*}, j\left(x-x^{*}\right)\right\rangle \geq 0 \forall x \in C$. This is equivalent to the dual VI of approximating $x^{*} \in C$ such that $\left\langle A x, j\left(x-x^{*}\right)\right\rangle \geq 0 \forall x \in C$.

Lemma 8 ([33]). Fix two bounded sequences $\left\{x_{n}\right\}$ and $\left\{w_{n}\right\}$ in a Banach space $E$ and set $\left\{\beta_{n}\right\}$ a sequence in $[0,1]$ with $1 \geq \limsup _{n \rightarrow \infty} \beta_{n} \geq \liminf _{n \rightarrow \infty} \beta_{n} \geq 0$. We suppose $x_{n+1}=\beta_{n}\left(x_{n}-w_{n}\right)+w_{n}, \forall n \geq 0$ and $\limsup _{n \rightarrow \infty}\left(\left\|w_{n+1}-w_{n}\right\|-\left\|x_{n+1}-x_{n}\right\|\right) \leq 0$. It follows $w_{n}-x_{n} \rightarrow 0$ as $n \rightarrow \infty$.

Lemma 9 ([34]). Assume that linear bounded operator $F$ is strongly positive on a smooth Banach space E. If $\bar{\gamma}>0$ and $\|F\|^{-1} \geq \rho>0$, then, $1-\rho \bar{\gamma} \geq\|I-\rho F\|$.

Lemma 10 ([34]). If Banach space $E$ is a both smooth and uniformly convex, then for $r>0$, there exists a convex, strictly increasing, continuous function $g:[0,2 r] \rightarrow \mathbf{R}, g(0)=0$ such that $\forall x, y \in\{x \in E:\|x\| \leq r\}$, $g(\|x-y\|)+2\langle x, j(y)\rangle \leq\|x\|^{2}+\|y\|^{2}$. 
Lemma 11 ([35]). Fix a convex nonempty and closed set $C$ in a reflexive Banach space E and set $T: C \rightarrow E$ a nonexpansive non-self-mapping. Suppose that E enjoys a weakly sequentially continuous duality mapping. If $\left\|T x_{n}-x_{n}\right\| \rightarrow 0$ as $n \rightarrow \infty$ and $x_{n} \rightarrow x$, then $x \in \operatorname{Fix}(T)$.

Lemma 12 ([36]). Let $\left\{a_{n}\right\}$ be a sequence in $[0,+\infty)$ such that $0 \leq a_{n+1} \leq v_{n}+s_{n} t_{n}+\left(1-s_{n}\right) a_{n} \forall n \geq 0$, where $\left\{s_{n}\right\},\left\{t_{n}\right\}$ and $\left\{v_{n}\right\}$ satisfy the assumptions: (i) $\limsup _{n \rightarrow \infty} t_{n} \leq 0$; (ii) $\left\{s_{n}\right\} \subset[0,1], \sum_{n=0}^{\infty} s_{n}=\infty$; (iii) $v_{n} \geq 0 \forall n \geq 0, \sum_{n=0}^{\infty} v_{n}<\infty$. Then $\lim _{n \rightarrow \infty} a_{n}=0$.

\section{Main Results}

In this part, we analyze a relaxed Mann implicit iteration method for approximating solutions of the GSVI (3) with the hierarchical variational inequality (HVI) constraint for countable many uniformly Lipschitzian pseudocontractions and an asymptotically nonexpansive mapping, which is in the intermediate sense in a real Banach space.

Theorem 1. Fix a convex nonempty and closed set $C$ in a 2-uniformly smooth and uniformly convex Banach space $E$. The space is assumed to enjoy a weakly sequentially continuous duality mapping. Let $\Pi_{C}: E \rightarrow C$ be a nonexpansive sunny retraction. Let the accretive mappings $B_{1}, B_{2}: C \rightarrow E$ be $\alpha$-inverse-strongly and $\beta$-inverse-strongly accretive. Let linear bounded operator $F: E \rightarrow E$ be strongly positive with coefficient $\bar{\gamma}>0$. Let $T: C \rightarrow C$ be uniformly continuous and asymptotically nonexpansive mapping in the intermediate sense, and $\left\{S_{n}\right\}_{n=0}^{\infty}$ be a countable family of $\ell$-uniformly Lipschitzian pseudocontractions on $C$ such that $\Omega:=\bigcap_{n=0}^{\infty} \operatorname{Fix}\left(S_{n}\right) \cap \operatorname{GSVI}\left(C, B_{1}, B_{2}\right) \cap \operatorname{Fix}(T) \neq \varnothing$ where $\operatorname{GSVI}\left(C, B_{1}, B_{2}\right)$ is the fixed-point set of the mapping $G:=\Pi_{C}\left(I-\mu_{1} B_{1}\right) \Pi_{C}\left(I-\mu_{2} B_{2}\right)$ with $0<\mu_{1}<\frac{\alpha}{\kappa^{2}}$ and $0<\mu_{2}<\frac{\beta}{\kappa^{2}}$. Suppose $\sum_{n=0}^{\infty} c_{n}<\infty$, where $c_{n}$ is defined by (2). For arbitrarily given $x_{0} \in C$, let $\left\{x_{n}\right\}$ be the sequence generated by

$$
\left\{\begin{array}{l}
x_{n+1}=\left(1-\beta_{n}\right) \Pi_{C}\left(T^{n} y_{n}-\alpha_{n} F T^{n} y_{n}\right)+\beta_{n} x_{n} \\
z_{n}=\Pi_{C}\left(u_{n}-\mu_{2} B_{2} u_{n}\right), \\
y_{n}=\Pi_{C}\left(z_{n}-\mu_{1} B_{1} z_{n}\right), \\
u_{n}=\gamma_{n} x_{n}+\left(1-\gamma_{n}\right) S_{n} u_{n}, \quad \forall n \geq 0
\end{array}\right.
$$

where $\left\{\alpha_{n}\right\},\left\{\beta_{n}\right\}$ and $\left\{\gamma_{n}\right\}$ are the real sequences in the interval $[0,1]$ satisfying:

(i) $\lim _{n \rightarrow \infty} \alpha_{n}=0$ and $\sum_{n=0}^{\infty} \alpha_{n}=\infty$;

(ii) $\limsup _{n \rightarrow \infty} \beta_{n}<1$ and $\liminf _{n \rightarrow \infty} \beta_{n}>0$;

(iii) $\lim \sup _{n \rightarrow \infty} \gamma_{n}<1, \liminf _{n \rightarrow \infty} \gamma_{n}>0$ and $\lim _{n \rightarrow \infty}\left|\gamma_{n}-\gamma_{n+1}\right|=0$.

Suppose $\sum_{n=0}^{\infty} \sup _{x \in D}\left\|S_{n} x-S_{n+1} x\right\|<\infty$, where $D$ is a bounded set in $C$, and let $S: C \rightarrow C$ be a noneslf mapping defined by $S x=\lim _{n \rightarrow \infty} S_{n} x$ for all $x \in C$, and assume that $\operatorname{Fix}(S)=\bigcap_{n=0}^{\infty} \operatorname{Fix}\left(S_{n}\right)$. Therefore, $\left\{x_{n}\right\}$ strongly converges to $x^{*} \in \Omega$ provided $\lim _{n \rightarrow \infty}\left\|T^{n} y_{n}-T^{n+1} y_{n}\right\|=0$. In this case,

(a) $x^{*} \in \Omega$ solves the VI: $\left\langle F\left(x^{*}\right), j\left(x^{*}-x\right)\right\rangle \leq 0, \forall x \in \Omega$;

(b) $\left(x^{*}, y^{*}\right)$ solves the GSVI (3) with $y^{*}=\Pi_{C}\left(x^{*}-\mu_{2} B_{2} x^{*}\right)$.

Proof. From $\lim _{n \rightarrow \infty} \alpha_{n}=0$, one may assume $\alpha_{n} \leq\|F\|^{-1} \forall n \geq 0$. By Lemma 9 we get $1-\alpha_{n} \bar{\gamma} \geq$ $\left\|I-\alpha_{n} F\right\|$. Since $1>\lim \sup _{n \rightarrow \infty} \gamma_{n} \geq \liminf _{n \rightarrow \infty} \gamma_{n}>0$, one supposes, without loss of generality, that $\left\{\gamma_{n}\right\} \subset[a, b] \subset(0,1)$ for some $a, b \in(0,1)$. Also, since $G: C \rightarrow C$ is defined as $G:=\Pi_{C}\left(\Pi_{C}(I-\right.$ $\left.\left.\mu_{2} B_{2}\right)-\mu_{1} B_{1} \Pi_{C}\left(I-\mu_{2} B_{2}\right)\right)$, where $0<\mu_{1} \kappa^{2} \leq \alpha$ and $0<\mu_{2} \kappa^{2} \leq \beta$, according to Lemma $5 G$ is nonexpansive. For each $n \geq 0$, one has a unique $u_{n} \in C$ such that

$$
u_{n}=\gamma_{n}\left(x_{n}-S_{n} u_{n}\right)+S_{n} u_{n}
$$

As a matter of fact, we consider $F_{n} x:=\gamma_{n}\left(x_{n}-S_{n} x\right)+S_{n} x \forall x \in C$. Since mapping $S_{n}: C \rightarrow C$ is a continuous pseudocontractive, one gets $\left(1-\gamma_{n}\right)\|x-y\|^{2} \geq\left\langle F_{n} x-F_{n} y, j(x-y)\right\rangle, \forall x, y \in C$. Also, from $\left\{\gamma_{n}\right\} \subset[a, b] \subset(0,1)$ we get $0<1-\gamma_{n}<1$ for all $n \geq 0$. Thus, $F_{n}$ is a continuous and strong 
pseudocontraction of $C$ into itself. By Proposition 2, we know that for each $n \geq 0$ there exists a unique element $u_{n} \in C$, satisfying (5). Therefore, it can be readily seen that the relaxed Mann implicit scheme (4) can be rewritten as

$$
\left\{\begin{array}{l}
u_{n}=\gamma_{n} x_{n}+\left(1-\gamma_{n}\right) S_{n} u_{n}, \\
y_{n}=G u_{n} \\
x_{n+1}=\left(1-\beta_{n}\right) \Pi_{C}\left(T^{n} y_{n}-\alpha_{n} F T^{n} y_{n}\right)+\beta_{n} x_{n}, \quad \forall n \geq 0
\end{array}\right.
$$

Next, we divide the rest of the proof into several steps.

Step 1. One proves that $\left\{x_{n}\right\},\left\{y_{n}\right\},\left\{z_{n}\right\},\left\{u_{n}\right\},\left\{T^{n} y_{n}\right\}$ and $\left\{F\left(T^{n} y_{n}\right)\right\}$ are bounded. Indeed, take an arbitrary $p \in \Omega=\bigcap_{n=0}^{\infty} \operatorname{Fix}\left(S_{n}\right) \cap \operatorname{GSVI}\left(C, B_{1}, B_{2}\right) \cap \operatorname{Fix}(T)$. So $S_{n} p=p, G p=p$ and $T p=p$. Since each $S_{n}$ is pseudocontractive on $C$, it follows that

$$
\left\|u_{n}-p\right\|^{2} \leq \gamma_{n}\left\|x_{n}-p\right\|\left\|p-u_{n}\right\|+\left(1-\gamma_{n}\right)\left\|u_{n}-p\right\|^{2},
$$

which hence yields

$$
\left\|u_{n}-p\right\| \leq\left\|x_{n}-p\right\|, \quad \forall n \geq 0
$$

Then we observe

$$
\left\|y_{n}-p\right\|=\left\|G u_{n}-p\right\| \leq\left\|x_{n}-p\right\| .
$$

Combining (6) and (8), we have

$$
\begin{aligned}
\left\|x_{n+1}-p\right\| & \leq\left(1-\beta_{n}\right)\left\|\Pi_{C}\left(I-\alpha_{n} F\right) T^{n} y_{n}-p\right\|+\beta_{n}\left\|x_{n}-p\right\| \\
& \leq\left(1-\beta_{n}\right)\left\|\left(I-\alpha_{n} F\right) T^{n} y_{n}-\left(I-\alpha_{n} F\right) p\right\|+\alpha_{n}\left(1-\beta_{n}\right)\|F(p)\|+\beta_{n}\left\|p-x_{n}\right\| \\
& \leq c_{n}+\left[1-\alpha_{n}\left(1-\beta_{n}\right) \bar{\gamma}\right]\left\|x_{n}-p\right\|+\alpha_{n}\left(1-\beta_{n}\right) \bar{\gamma} \frac{\|F(p)\|}{\bar{\gamma}} \\
& \leq c_{n}+\max \left\{\left\|p-x_{n}\right\|, \frac{\|F(p)\|}{\bar{\gamma}}\right\} .
\end{aligned}
$$

By induction, we obtain $0 \leq\left\|x_{n}-p\right\| \leq \sum_{n=0}^{\infty} c_{n}+\max \left\{\frac{\|F(p)\|}{\bar{\gamma}},\left\|x_{0}-p\right\|\right\}, \forall n \geq 0$. It directly follows that $\left\{x_{n}\right\}$ is a bounded vector sequence. This is also true for the sequences $\left\{y_{n}\right\},\left\{u_{n}\right\},\left\{T^{n} y_{n}\right\}$ and $\left\{F\left(T^{n} y_{n}\right)\right\}$ (due to (2), (7), (8) and the Lipschitz continuity). Since $\left\{S_{n}\right\}$ is $\ell$-uniformly Lipschitzian, we easily know that $\left\|S_{n} u_{n}\right\| \leq\left\|S_{n} u_{n}-p\right\|+\|p\| \leq \ell\left\|u_{n}-p\right\|+\|p\|$, which implies that $\left\{S_{n} u_{n}\right\}$ is bounded. Additionally, by using Lemma 3 and $p \in \Omega \subset \operatorname{GSVI}\left(C, B_{1}, B_{2}\right)$ we know that $(p, q)$ solves the GSVI (3) where $q=\Pi_{C}\left(I-\mu_{2} B_{2}\right) p$. Since $z_{n}=\Pi_{C}\left(I-\mu_{2} B_{2}\right) u_{n} \forall n \geq 0$, by Lemma 4 we get $\left\|z_{n}\right\| \leq\left\|\Pi_{C}\left(I-\mu_{2} B_{2}\right) u_{n}-\Pi_{C}\left(I-\mu_{2} B_{2}\right) p\right\|+\|q\| \leq\left\|u_{n}-p\right\|+\|q\|$. This shows that $\left\{z_{n}\right\}$ is bounded.

Step 2. We prove $\left\|x_{n}-x_{n+1}\right\| \rightarrow 0$ and $\left\|y_{n}-y_{n+1}\right\| \rightarrow 0$ as $n \rightarrow \infty$. Indeed, one put $x_{n+1}=$ $\left(1-\beta_{n}\right) w_{n}+\beta_{n} x_{n} \forall n \geq 0$ with $w_{n}=\Pi_{C}\left(I-\alpha_{n} F\right) T^{n} y_{n}$. From (6) and (2) we get

$$
\begin{aligned}
& \left\|w_{n+1}-w_{n}\right\| \leq\left\|\left(I-\alpha_{n+1} F\right) T^{n+1} y_{n+1}-\left(I-\alpha_{n} F\right) T^{n} y_{n}\right\| \\
& \leq\left\|T^{n+1} y_{n+1}-T^{n+1} y_{n}\right\|+\alpha_{n+1}\left\|F\left(T^{n+1} y_{n+1}\right)\right\|+\left\|T^{n+1} y_{n}-T^{n} y_{n}\right\|+\alpha_{n}\left\|F\left(T^{n} y_{n}\right)\right\| \\
& \leq\left\|y_{n+1}-y_{n}\right\|+c_{n+1}+\left\|T^{n+1} y_{n}-T^{n} y_{n}\right\|+\alpha_{n+1}\left\|F\left(T^{n+1} y_{n+1}\right)\right\|+\alpha_{n}\left\|F\left(T^{n} y_{n}\right)\right\| \\
& \leq\left\|u_{n+1}-u_{n}\right\|+c_{n+1}+\alpha_{n+1}\left\|F\left(T^{n+1} y_{n+1}\right)\right\|+\left\|T^{n+1} y_{n}-T^{n} y_{n}\right\|+\alpha_{n}\left\|F\left(T^{n} y_{n}\right)\right\| .
\end{aligned}
$$

Since simple calculations show that

$$
\begin{aligned}
& \left\|u_{n}-u_{n+1}\right\|^{2} \\
& =\gamma_{n+1}\left\langle u_{n+1}-u_{n}, x_{n+1}-x_{n}\right\rangle+\left(1-\gamma_{n+1}\right)\left[\left\langle S_{n+1} u_{n+1}-S_{n} u_{n+1}, u_{n+1}-u_{n}\right\rangle\right. \\
& \left.\quad+\left\langle S_{n} u_{n+1}-S_{n} u_{n}, u_{n+1}-u_{n}\right\rangle\right]+\left(\gamma_{n+1}-\gamma_{n}\right)\left\langle x_{n}-S_{n} u_{n}, u_{n+1}-u_{n}\right\rangle \\
& \leq \gamma_{n+1}\left\|x_{n}-x_{n+1}\right\|\left\|u_{n+1}-u_{n}\right\|+\left(1-\gamma_{n+1}\right)\left[\left\|S_{n+1} u_{n+1}-S_{n} u_{n+1}\right\|\left\|u_{n+1}-u_{n}\right\|\right. \\
& \left.\quad+\left\|u_{n+1}-u_{n}\right\|^{2}\right]+\left|\gamma_{n}-\gamma_{n+1}\right|\left\|S_{n} u_{n}-x_{n}\right\|\left\|u_{n}-u_{n+1}\right\|
\end{aligned}
$$


it follows from (10) that

$$
\begin{aligned}
\left\|u_{n+1}-u_{n}\right\| & \leq\left\|x_{n}-x_{n+1}\right\|+\left|\gamma_{n}-\gamma_{n+1}\right| \frac{\left\|x_{n}-S_{n} u_{n}\right\|}{\gamma_{n+1}}+\frac{1-\gamma_{n+1}}{\gamma_{n+1}}\left\|S_{n} u_{n+1}-S_{n+1} u_{n+1}\right\| \\
& \leq \frac{1}{a}\left\|S_{n+1} u_{n+1}-S_{n} u_{n+1}\right\|+\left|\gamma_{n+1}-\gamma_{n}\right| \frac{\left\|x_{n}-S_{n} u_{n}\right\|}{a}+\left\|x_{n}-x_{n+1}\right\| .
\end{aligned}
$$

Also, since $D:=\left\{u_{n}: n \geq 0\right\} \subset C$ is bounded, one gets $\sum_{n=0}^{\infty} \sup _{x \in D}\left\|S_{n+1} x-S_{n} x\right\|<\infty$. Hence we have

$$
\sum_{n=0}^{\infty}\left\|S_{n} u_{n+1}-S_{n+1} u_{n+1}\right\|<\infty
$$

Therefore, from (9) and (11) we deduce that

$$
\begin{aligned}
a\left\|w_{n}-w_{n+1}\right\| \leq \leq & a\left\|x_{n}-x_{n+1}\right\|+\left\|S_{n+1} u_{n+1}-S_{n} u_{n+1}\right\|+a\left|\gamma_{n+1}-\gamma_{n}\right| \frac{\left\|x_{n}-S_{n} u_{n}\right\|}{a} \\
& +a c_{n+1}+\left\|T^{n+1} y_{n}-T^{n} y_{n}\right\|+a \alpha_{n+1}\left\|F\left(T^{n+1} y_{n+1}\right)\right\|+a \alpha_{n}\left\|F\left(T^{n} y_{n}\right)\right\|,
\end{aligned}
$$

which immediately attains

$$
\begin{aligned}
& \left\|w_{n}-w_{n+1}\right\|-\left\|x_{n}-x_{n+1}\right\| \\
& \leq \frac{1}{a}\left\|S_{n+1} u_{n+1}-S_{n} u_{n+1}\right\|+\left|\gamma_{n+1}-\gamma_{n}\right| \frac{\left\|x_{n}-S_{n} u_{n}\right\|}{a}+c_{n+1} \\
& \quad+\left\|T^{n+1} y_{n}-T^{n} y_{n}\right\|+\alpha_{n}\left\|F\left(T^{n} y_{n}\right)\right\|+\alpha_{n+1}\left\|F\left(T^{n+1} y_{n+1}\right)\right\| .
\end{aligned}
$$

Since $\lim _{n \rightarrow \infty} c_{n}=0$ (due to (2)) and $\lim _{n \rightarrow \infty}\left\|T^{n} y_{n}-T^{n+1} y_{n}\right\|=0$ (due to the assumption), from (12) and conditions (i), (iii) it follows that $\limsup _{n \rightarrow \infty}\left(\left\|w_{n+1}-w_{n}\right\|-\left\|x_{n+1}-x_{n}\right\|\right) \leq 0$. Hence, by condition (ii) and Lemma 8 , we get $\lim _{n \rightarrow \infty}\left\|w_{n}-x_{n}\right\|=0$. Consequently,

$$
\lim _{n \rightarrow \infty}\left\|x_{n}-x_{n+1}\right\|=\lim _{n \rightarrow \infty}\left(1-\beta_{n}\right)\left\|x_{n}-w_{n}\right\|=0 .
$$

Again from (6) and (11) we conclude that

$$
\begin{aligned}
& a\left\|y_{n+1}-y_{n}\right\|=a\left\|G u_{n+1}-G u_{n}\right\| \leq a\left\|u_{n}-u_{n+1}\right\| \\
& \leq\left\|S_{n+1} u_{n+1}-S_{n} u_{n+1}\right\|+a\left\|x_{n+1}-x_{n}\right\|+a\left|\gamma_{n+1}-\gamma_{n}\right|\left\|x_{n}-S_{n} u_{n}\right\| \rightarrow 0 \quad(n \rightarrow \infty) .
\end{aligned}
$$

Step 3. One proves $\lim _{n \rightarrow \infty}\left\|x_{n}-G x_{n}\right\|=0$. Indeed, since $w_{n}=\Pi_{C}\left(I-\alpha_{n} F\right) T^{n} y_{n}$, according to Proposition 3, we have that for each $p \in \Omega$,

$$
\left\langle\Pi_{C}\left(I-\alpha_{n} F\right) T^{n} y_{n}-\left(I-\alpha_{n} F\right) T^{n} y_{n}, j\left(p-w_{n}\right)\right\rangle \geq 0 .
$$

From (16), we have

$$
\begin{aligned}
2\left\|w_{n}-p\right\|^{2}= & 2\left\langle\Pi_{C}\left(I-\alpha_{n} F\right) T^{n} y_{n}-\left(I-\alpha_{n} F\right) T^{n} y_{n}, j\left(w_{n}-p\right)\right\rangle \\
& +2\left\langle\left(I-\alpha_{n} F\right) T^{n} y_{n}-p, j\left(w_{n}-p\right)\right\rangle \\
\leq & 2\left\langle\left(I-\alpha_{n} F\right)\left(T^{n} y_{n}-p\right), j\left(w_{n}-p\right)\right\rangle+2 \alpha_{n}\left\langle F(p), j\left(p-w_{n}\right)\right\rangle \\
\leq & \left(1-\alpha_{n} \bar{\gamma}\right)^{2}\left\|T^{n} y_{n}-p\right\|^{2}+\left\|p-w_{n}\right\|^{2}+2 \alpha_{n}\left\langle F(p), j\left(p-w_{n}\right)\right\rangle .
\end{aligned}
$$

It follows from (2) that

$$
\begin{aligned}
\left\|w_{n}-p\right\|^{2} & \leq 2 \alpha_{n}\left\langle F(p), j\left(p-w_{n}\right)\right\rangle+\left(1-\alpha_{n} \bar{\gamma}\right)^{2}\left\|T^{n} y_{n}-p\right\|^{2} \\
& \leq c_{n}\left(2\left\|y_{n}-p\right\|+c_{n}\right)+\left(1-\alpha_{n} \bar{\gamma}\right)\left\|p-y_{n}\right\|^{2}+2 \alpha_{n}\left\langle F(p), j\left(p-w_{n}\right)\right\rangle .
\end{aligned}
$$

From (4) and (17), we get

$$
\begin{aligned}
\left\|x_{n+1}-p\right\|^{2} \leq & \left(1-\beta_{n}\right)\left(1-\alpha_{n} \bar{\gamma}\right)\left\|y_{n}-p\right\|^{2}+\beta_{n}\left\|x_{n}-p\right\|^{2} \\
& +c_{n}\left(2\left\|y_{n}-p\right\|+c_{n}\right)+2 \alpha_{n}\left(1-\beta_{n}\right)\left\langle F(p), j\left(p-w_{n}\right)\right\rangle .
\end{aligned}
$$


We now note that $q=\Pi_{C}\left(p-\mu_{2} B_{2} p\right), z_{n}=\Pi_{C}\left(u_{n}-\mu_{2} B_{2} u_{n}\right)$ and $y_{n}=\Pi_{C}\left(z_{n}-\mu_{1} B_{1} z_{n}\right)$. Then $y_{n}=G u_{n}$. By using the Lemma 4 ,

$$
\left\|z_{n}-q\right\|^{2} \leq\left\|u_{n}-p-\mu_{2}\left(B_{2} u_{n}-B_{2} p\right)\right\|^{2} \leq\left\|u_{n}-p\right\|^{2}-2 \mu_{2}\left(\beta-\kappa^{2} \mu_{2}\right)\left\|B_{2} u_{n}-B_{2} p\right\|^{2},
$$

and

$$
\left\|y_{n}-p\right\|^{2} \leq\left\|z_{n}-q-\mu_{1}\left(B_{1} z_{n}-B_{1} q\right)\right\|^{2} \leq\left\|z_{n}-q\right\|^{2}-2 \mu_{1}\left(\alpha-\kappa^{2} \mu_{1}\right)\left\|B_{1} z_{n}-B_{1} q\right\|^{2} .
$$

Substituting (19) for (20), we obtain from (7) that

$$
\left\|y_{n}-p\right\|^{2}+2 \mu_{2}\left(\beta-\kappa^{2} \mu_{2}\right)\left\|B_{2} u_{n}-B_{2} p\right\|^{2}+2 \mu_{1}\left(\alpha-\kappa^{2} \mu_{1}\right)\left\|B_{1} z_{n}-B_{1} q\right\|^{2} \leq\left\|x_{n}-p\right\|^{2} .
$$

Combining (18) and (21), we get

$$
\begin{aligned}
& \left\|p-x_{n+1}\right\|^{2} \\
& \leq\left(1-\beta_{n}\right)\left(1-\alpha_{n} \bar{\gamma}\right)\left[\left\|x_{n}-p\right\|^{2}-2 \mu_{2}\left(\beta-\kappa^{2} \mu_{2}\right)\left\|B_{2} u_{n}-B_{2} p\right\|^{2}+\beta_{n}\left\|p-x_{n}\right\|^{2}\right. \\
& \left.\quad+2 \mu_{1}\left(\kappa^{2} \mu_{1}-\alpha\right)\left\|B_{1} z_{n}-B_{1} q\right\|^{2}\right]+2 \alpha_{n}\|F(p)\|\left\|p-w_{n}\right\|+c_{n}\left(2\left\|y_{n}-p\right\|+c_{n}\right) \\
& =\left[1-\alpha_{n}\left(1-\beta_{n}\right) \bar{\gamma}\right]\left\|p-x_{n}\right\|^{2}-2\left(1-\beta_{n}\right)\left(1-\alpha_{n} \bar{\gamma}\right)\left[\mu_{2}\left(\beta-\kappa^{2} \mu_{2}\right)\left\|B_{2} u_{n}-B_{2} p\right\|^{2}\right. \\
& \left.\quad+\mu_{1}\left(\alpha-\kappa^{2} \mu_{1}\right)\left\|B_{1} z_{n}-B_{1} q\right\|^{2}\right]+c_{n}\left(2\left\|y_{n}-p\right\|+c_{n}\right)+2 \alpha_{n}\|F(p)\|\left\|p-w_{n}\right\|,
\end{aligned}
$$

which immediately yields

$$
\begin{aligned}
& 2\left(1-\beta_{n}\right)\left(\alpha_{n} \bar{\gamma}-1\right)\left[\mu_{2}\left(\beta-\kappa^{2} \mu_{2}\right)\left\|B_{2} u_{n}-B_{2} p\right\|^{2}+\mu_{1}\left(\alpha-\kappa^{2} \mu_{1}\right)\left\|B_{1} z_{n}-B_{1} q\right\|^{2}\right] \\
& \geq\left\|x_{n+1}-p\right\|^{2}-\left\|x_{n}-p\right\|^{2}-c_{n}\left(2\left\|y_{n}-p\right\|+c_{n}\right)-2 \alpha_{n}\|F(p)\|\left\|p-w_{n}\right\| \\
& \geq-c_{n}\left(2\left\|y_{n}-p\right\|+c_{n}\right)-2 \alpha_{n}\|F(p)\|\left\|p-w_{n}\right\|-\left(p-\left\|x_{n}\right\|+\left\|p-x_{n+1}\right\|\right)\left\|x_{n}-x_{n+1}\right\| .
\end{aligned}
$$

Since $\liminf \operatorname{in}_{n \rightarrow \infty}\left(1-\beta_{n}\right)>0$ (due to condition (ii)), $\mu_{1} \in\left(0, \frac{\alpha}{\kappa^{2}}\right), \mu_{2} \in\left(0, \frac{\beta}{\kappa^{2}}\right), \lim _{n \rightarrow \infty} c_{n}=0$ and $\lim _{n \rightarrow \infty} \alpha_{n}=0$, we obtain from (14) that

$$
\lim _{n \rightarrow \infty}\left\|B_{2} u_{n}-B_{2} p\right\|=\lim _{n \rightarrow \infty}\left\|B_{1} z_{n}-B_{1} q\right\|=0 .
$$

On the other hand, from Lemma 10 and Proposition 3 we have

$$
\begin{array}{rl}
2\left\|q-z_{n}\right\|^{2} & 2 \leq\left\langle j\left(z_{n}-q\right), u_{n}-\mu_{2} B_{2} u_{n}-\left(p-\mu_{2} B_{2} p\right)\right\rangle \\
& =2 \mu_{2}\left\langle B_{2} p-B_{2} u_{n}, j\left(z_{n}-q\right)\right\rangle+\left\langle j\left(z_{n}-q\right), u_{n}-p\right\rangle \\
& \leq\left[\left\|u_{n}-p\right\|^{2}+\left\|z_{n}-q\right\|^{2}-g_{1}\left(\left\|u_{n}-z_{n}-(p-q)\right\|\right)\right]+2 \mu_{2}\left\|B_{2} p-B_{2} u_{n}\right\|\left\|z_{n}-q\right\|,
\end{array}
$$

which implies that

$$
\left\|z_{n}-q\right\|^{2}+g_{1}\left(\left\|u_{n}-z_{n}-(p-q)\right\|\right) \leq\left\|u_{n}-p\right\|^{2}+2 \mu_{2}\left\|B_{2} p-B_{2} u_{n}\right\|\left\|z_{n}-q\right\| .
$$

In the same manner, one reaches

$$
\left\|y_{n}-p\right\|^{2}+g_{2}\left(\left\|z_{n}-y_{n}+(p-q)\right\|\right) \leq\left\|z_{n}-q\right\|^{2}+2 \mu_{1}\left\|B_{1} q-B_{1} z_{n}\right\|\left\|y_{n}-p\right\| .
$$

Substituting (23) for (24), we deduce from (7) that

$$
\begin{aligned}
& \left\|y_{n}-p\right\|^{2}+g_{1}\left(\left\|u_{n}-z_{n}-(p-q)\right\|\right)+g_{2}\left(\left\|z_{n}-y_{n}+(p-q)\right\|\right) \\
& \leq\left\|x_{n}-p\right\|^{2}+2 \mu_{1}\left\|B_{1} q-B_{1} z_{n}\right\|\left\|y_{n}-p\right\|+2 \mu_{2}\left\|B_{2} p-B_{2} u_{n}\right\|\left\|z_{n}-q\right\| .
\end{aligned}
$$


Combining (18) and (25), we have

$$
\begin{aligned}
\left\|x_{n+1}-p\right\|^{2} \leq & \left(1-\beta_{n}\right)\left(1-\alpha_{n} \bar{\gamma}\right)\left[\left\|x_{n}-p\right\|^{2}+\beta_{n}\left\|x_{n}-p\right\|^{2}\right. \\
& -g_{1}\left(\left\|u_{n}-z_{n}-(p-q)\right\|\right)-g_{2}\left(\left\|z_{n}-y_{n}+(p-q)\right\|\right) \\
& \left.+2 \mu_{1}\left\|B_{1} q-B_{1} z_{n}\right\|\left\|y_{n}-p\right\|+2 \mu_{2}\left\|B_{2} p-B_{2} u_{n}\right\|\left\|z_{n}-q\right\|\right] \\
& +c_{n}\left(2\left\|y_{n}-p\right\|+c_{n}\right)+2 \alpha_{n}\|F(p)\|\left\|p-w_{n}\right\| \\
= & {\left[1-\alpha_{n}\left(1-\beta_{n}\right) \bar{\gamma}\right]\left\|x_{n}-p\right\|^{2}-\left(1-\beta_{n}\right)\left(1-\alpha_{n} \bar{\gamma}\right) \times } \\
& \times\left[g_{1}\left(\left\|u_{n}-z_{n}-(p-q)\right\|\right)+g_{2}\left(\left\|z_{n}-y_{n}+(p-q)\right\|\right)\right] \\
& +2 \mu_{2}\left\|B_{2} p-B_{2} u_{n}\right\|\left\|z_{n}-q\right\|+2 \mu_{1}\left\|B_{1} q-B_{1} z_{n}\right\|\left\|y_{n}-p\right\| \\
& +c_{n}\left(2\left\|y_{n}-p\right\|+c_{n}\right)+2 \alpha_{n}\|F(p)\|\left\|p-w_{n}\right\|,
\end{aligned}
$$

which hence yields

$$
\begin{aligned}
& \left(1-\beta_{n}\right)\left(1-\alpha_{n} \bar{\gamma}\right)\left[g_{1}\left(\left\|u_{n}-z_{n}-(p-q)\right\|\right)+g_{2}\left(\left\|z_{n}-y_{n}+(p-q)\right\|\right)\right] \\
& \leq\left\|p-x_{n}\right\|^{2}-\left\|p-x_{n+1}\right\|^{2}+2 \mu_{2}\left\|B_{2} p-B_{2} u_{n}\right\|\left\|z_{n}-q\right\| \\
& \quad+2 \mu_{1}\left\|B_{1} q-B_{1} z_{n}\right\|\left\|y_{n}-p\right\|+c_{n}\left(2\left\|y_{n}-p\right\|+c_{n}\right)+2 \alpha_{n}\|F(p)\|\left\|p-w_{n}\right\| \\
& \leq\left(\left\|p-x_{n}\right\|+\left\|p-x_{n+1}\right\|\right)\left\|x_{n}-x_{n+1}\right\|+2 \mu_{2}\left\|B_{2} p-B_{2} u_{n}\right\|\left\|z_{n}-q\right\| \\
& \quad+c_{n}\left(2\left\|y_{n}-p\right\|+c_{n}\right)+2 \alpha_{n}\|F(p)\|\left\|p-w_{n}\right\|+2 \mu_{1}\left\|B_{1} q-B_{1} z_{n}\right\|\left\|y_{n}-p\right\| .
\end{aligned}
$$

Since $\liminf _{n \rightarrow \infty}\left(1-\beta_{n}\right)>0$ (due to condition (ii)), $\lim _{n \rightarrow \infty} c_{n}=0$ and $\lim _{n \rightarrow \infty} \alpha_{n}=0$, we conclude from (14) and (22) that $g_{1}\left(\left\|u_{n}-z_{n}-(p-q)\right\|\right) \rightarrow 0$ as $n \rightarrow \infty$ and $g_{2}\left(\left\|z_{n}-y_{n}+(p-q)\right\|\right) \rightarrow 0$ as $n \rightarrow \infty$. Using the properties of $g_{1}$ and $g_{2}$, we obtain

$$
\lim _{n \rightarrow \infty}\left\|u_{n}-z_{n}-(p-q)\right\|=0 \quad \text { and } \quad \lim _{n \rightarrow \infty}\left\|z_{n}-y_{n}+(p-q)\right\|=0 .
$$

It follows that

$$
\left\|u_{n}-G u_{n}\right\|=\left\|u_{n}-y_{n}\right\| \leq\left\|z_{n}-u_{n}-(q-p)\right\|+\left\|z_{n}-y_{n}+(p-q)\right\| \rightarrow 0 \quad(n \rightarrow \infty) .
$$

Also, according to (5) we have $\left\|u_{n}-p\right\|^{2} \leq \gamma_{n}\left\langle x_{n}-p, j\left(u_{n}-p\right)\right\rangle+\left\|u_{n}-p\right\|^{2}\left(1-\gamma_{n}\right)$, which together with Lemma 10, yields

$$
2\left\|u_{n}-p\right\|^{2} \leq 2\left\langle x_{n}-p, j\left(u_{n}-p\right)\right\rangle \leq\left\|p-x_{n}\right\|^{2}+\left\|p-u_{n}\right\|^{2}-g\left(\left\|x_{n}-u_{n}\right\|\right) .
$$

Therefore, we get $\left\|p-y_{n}\right\|^{2} \leq\left\|p-u_{n}\right\|^{2} \leq\left\|p-x_{n}\right\|^{2}-g\left(\left\|x_{n}-u_{n}\right\|\right)$, which together with (18), yields

$$
\begin{aligned}
\left\|x_{n+1}-p\right\|^{2} \leq & \beta_{n}\left\|x_{n}-p\right\|^{2}+\left(1-\beta_{n}\right)\left(1-\alpha_{n} \bar{\gamma}\right)\left[\left\|x_{n}-p\right\|^{2}-g\left(\left\|x_{n}-u_{n}\right\|\right)\right] \\
& +c_{n}\left(2\left\|y_{n}-p\right\|+c_{n}\right)+2 \alpha_{n}\left(1-\beta_{n}\right)\left\langle F(p), j\left(p-w_{n}\right)\right\rangle \\
\leq & {\left[1-\alpha_{n}\left(1-\beta_{n}\right) \bar{\gamma}\right]\left\|x_{n}-p\right\|^{2}-\left(1-\beta_{n}\right)\left(1-\alpha_{n} \bar{\gamma}\right) g\left(\left\|x_{n}-u_{n}\right\|\right) } \\
& +c_{n}\left(2\left\|y_{n}-p\right\|+c_{n}\right)+2 \alpha_{n}\|F(p)\|\left\|p-w_{n}\right\| .
\end{aligned}
$$

Thus, we have

$$
\begin{aligned}
& \left(1-\beta_{n}\right)\left(1-\alpha_{n} \bar{\gamma}\right) g\left(\left\|x_{n}-u_{n}\right\|\right) \\
& \leq\left\|p-x_{n}\right\|^{2}+c_{n}\left(2\left\|p-y_{n}\right\|+c_{n}\right)+2 \alpha_{n}\|F(p)\|\left\|p-w_{n}\right\|-\left\|x_{n+1}-p\right\|^{2} \\
& \leq\left(\left\|p-x_{n}\right\|+\left\|p-x_{n+1}\right\|\right)\left\|x_{n}-x_{n+1}\right\|+2 \alpha_{n}\|F(p)\|\left\|p-w_{n}\right\|+c_{n}\left(2\left\|y_{n}-p\right\|+c_{n}\right) .
\end{aligned}
$$

Since $\liminf \operatorname{in}_{n \rightarrow \infty}\left(1-\beta_{n}\right)>0$ (due to condition (ii)), $\lim _{n \rightarrow \infty} c_{n}=0$ and $\lim _{n \rightarrow \infty} \alpha_{n}=0$, we obtain from (14) that $\lim _{n \rightarrow \infty} g\left(\left\|x_{n}-u_{n}\right\|\right)=0$. We obtain

$$
\lim _{n \rightarrow \infty}\left\|x_{n}-u_{n}\right\|=0
$$


From (27) and (28) it follows that $\left\|x_{n}-y_{n}\right\| \leq\left\|x_{n}-u_{n}\right\|+\left\|u_{n}-G u_{n}\right\| \rightarrow 0$, and

$$
\left\|x_{n}-G x_{n}\right\| \leq\left\|x_{n}-y_{n}\right\|+\left\|G u_{n}-G x_{n}\right\| \leq\left\|x_{n}-y_{n}\right\|+\left\|u_{n}-x_{n}\right\| \rightarrow 0 \quad(n \rightarrow \infty) .
$$

Step 4. One proves that $\left\|T x_{n}-x_{n}\right\| \rightarrow 0$ and $\left\|S_{n} x_{n}-x_{n}\right\| \rightarrow 0$ as $n \rightarrow \infty$. By (5) and (28), we obtain that

$$
0 \leq\left\|S_{n} u_{n}-u_{n}\right\|=\frac{\gamma_{n}}{1-\gamma_{n}}\left\|x_{n}-u_{n}\right\| \leq \frac{b}{1-b}\left\|x_{n}-u_{n}\right\| \rightarrow 0 \quad(n \rightarrow \infty) .
$$

Since $\left\{S_{n}\right\}_{n=0}^{\infty}: C \rightarrow C$ is $\ell$-uniformly Lipschitzian, we deduce from (28) and (30) that

$$
\begin{aligned}
\left\|S_{n} x_{n}-x_{n}\right\| & \leq\left\|S_{n} x_{n}-S_{n} u_{n}\right\|+\left\|S_{n} u_{n}-u_{n}\right\|+\left\|u_{n}-x_{n}\right\| \\
& \leq(\ell+1)\left\|x_{n}-u_{n}\right\|+\left\|S_{n} u_{n}-u_{n}\right\| \rightarrow 0 \quad(n \rightarrow \infty) .
\end{aligned}
$$

Since $1 \geq \limsup \operatorname{su}_{n \rightarrow \infty} \beta_{n}$ and $\liminf _{n \rightarrow \infty} \beta_{n} \geq 0$, One supposes, without loss of generality, that $\left\{\beta_{n}\right\} \subset[c, d] \subset(0,1)$ for some $c, d \in(0,1)$. From $x_{n+1}=\beta_{n} x_{n}+\left(1-\beta_{n}\right) \Pi_{C}\left(I-\alpha_{n} F\right) T^{n} y_{n}$, we obtain $\left\|x_{n}-T^{n} y_{n}\right\| \leq\left\|x_{n}-x_{n+1}\right\|+\left\|x_{n+1}-T^{n} y_{n}\right\| \leq\left\|x_{n}-x_{n+1}\right\|+\beta_{n}\left\|x_{n}-T^{n} y_{n}\right\|+\left(1-\beta_{n}\right) \alpha_{n}\left\|F\left(T^{n} y_{n}\right)\right\|$. Then we have $(1-d)\left\|x_{n}-T^{n} y_{n}\right\| \leq\left\|x_{n}-x_{n+1}\right\|+(1-d) \alpha_{n}\left\|F\left(T^{n} y_{n}\right)\right\|$. Hence we get

$$
\left\|y_{n}-T^{n} y_{n}\right\| \leq\left\|y_{n}-x_{n}\right\|+\left\|x_{n}-T^{n} y_{n}\right\| \leq\left\|y_{n}-x_{n}\right\|+\frac{1}{1-d}\left\|x_{n}-x_{n+1}\right\|+\alpha_{n}\left\|F\left(T^{n} y_{n}\right)\right\| .
$$

Consequently, from (14), (29) and $\lim _{n \rightarrow \infty} \alpha_{n}=0$, it follows that

$$
\lim _{n \rightarrow \infty}\left\|x_{n}-T^{n} y_{n}\right\|=0 \quad \text { and } \quad \lim _{n \rightarrow \infty}\left\|y_{n}-T^{n} y_{n}\right\|=0 .
$$

We also note that $\left\|y_{n}-T y_{n}\right\| \leq\left\|y_{n}-T^{n} y_{n}\right\|+\left\|T^{n} y_{n}-T^{n+1} y_{n}\right\|+\left\|T^{n+1} y_{n}-T y_{n}\right\|$. By the assumption $\lim _{n \rightarrow \infty}\left\|T^{n+1} y_{n}-T^{n} y_{n}\right\|=0$, (32) and the condition that $T: C \rightarrow C$ is uniformly continuous, we get

$$
\lim _{n \rightarrow \infty}\left\|y_{n}-T y_{n}\right\|=0
$$

In addition, noticing that $\left\|x_{n}-T x_{n}\right\| \leq\left\|x_{n}-y_{n}\right\|+\left\|T y_{n}-T x_{n}\right\|+\left\|y_{n}-T y_{n}\right\|$, we deduce from (29), (33) and the uniform continuity of $T$ that

$$
\lim _{n \rightarrow \infty}\left\|x_{n}-T x_{n}\right\|=0
$$

Step 5. We claim that $\left\|x_{n}-\bar{S} x_{n}\right\| \rightarrow 0$ as $n \rightarrow \infty$ where $\bar{S}:=(I+I-S)^{-1}$. Indeed, we first observe that for all $x, y \in C, \lim _{n \rightarrow \infty}\left\|S_{n} x-S x\right\|=0$ and $\lim _{n \rightarrow \infty}\left\|S_{n} y-S y\right\|=0$. Since each $S_{n}$ is pseudocontractive, we get $\langle S x-S y, j(x-y)\rangle=\lim _{n \rightarrow \infty}\left\langle S_{n} x-S_{n} y, j(x-y)\right\rangle \leq\|x-y\|^{2}$. Thus, $S$ is pseudocontractive. Also, since $\left\{S_{n}\right\}_{n=0}^{\infty}$ is $\ell$-uniformly Lipschitzian on $C$, we get $\|S x-S y\|=\lim _{n \rightarrow \infty}\left\|S_{n} x-S_{n} y\right\| \leq \ell\|x-y\| \forall x, y \in C$. This means that $S$ is $\ell$-Lipschitzian. Noticing the boundedness of $\left\{x_{n}\right\}$ and putting $D:=\overline{\operatorname{conv}}\left\{x_{n}: n \geq 0\right\}$ (the closure of convex hull of the set $\left.\left\{x_{n}: n \geq 0\right\}\right)$, we have $\sum_{n=1}^{\infty} \sup _{x \in D}\left\|S_{n} x-S_{n-1} x\right\|<\infty$. Therefore, by Proposition 1 we get $\lim _{n \rightarrow \infty} \sup _{x \in D}\left\|S_{n} x-S x\right\|=0$, which immediately yields

$$
\lim _{n \rightarrow \infty}\left\|S_{n} x_{n}-S x_{n}\right\|=0
$$

Thus, combining (31) and (35) implies

$$
0 \leq\left\|x_{n}-S x_{n}\right\| \leq\left\|x_{n}-S_{n} x_{n}\right\|+\left\|S_{n} x_{n}-S x_{n}\right\| \rightarrow 0 \quad(n \rightarrow \infty) .
$$


Next, putting $\bar{S}:=(2 I-S)^{-1}$, one sees that $\bar{S}$ is nonexpansive, and $\operatorname{Fix}(\bar{S})=\operatorname{Fix}(S)=$ $\bigcap_{n=0}^{\infty} \operatorname{Fix}\left(S_{n}\right)$ (see [16]).

From (36) it follows that

$$
\begin{aligned}
\left\|x_{n}-\bar{S} x_{n}\right\| & =\left\|\overline{S S}^{-1} x_{n}-\bar{S} x_{n}\right\| \leq\left\|\bar{S}^{-1} x_{n}-x_{n}\right\| \\
& =\left\|(2 I-S) x_{n}-x_{n}\right\|=\left\|x_{n}-S x_{n}\right\| \rightarrow 0 \quad(n \rightarrow \infty) .
\end{aligned}
$$

Step 6. One proves that

$$
\limsup _{n \rightarrow \infty}\left\langle F\left(x^{*}\right), j\left(x^{*}-w_{n}\right)\right\rangle \leq 0,
$$

where $x^{*}=\Pi_{\Omega}(I-\rho F)\left(x^{*}\right)$ with $\rho \in\left(0,\|F\|^{-1}\right)$. There is a sequence $\left\{w_{n_{i}}\right\}$ such that $\lim \sup _{n \rightarrow \infty}\left\langle F\left(x^{*}\right), j\left(x^{*}-w_{n}\right)\right\rangle=\lim _{i \rightarrow \infty}\left\langle F\left(x^{*}\right), j\left(x^{*}-w_{n_{i}}\right)\right\rangle$. Now we show that $\Pi_{\Omega}(I-\rho F)$ is a contraction. For all $x, y \in C$, we get by Lemma 9

$$
\left\|\Pi_{\Omega}(I-\rho F)(x)-\Pi_{\Omega}(I-\rho F)(y)\right\| \leq\|(I-\rho F)(x)-(I-\rho F)(y)\| \leq(1-\bar{\gamma} \rho)\|x-y\| .
$$

Since $0<\rho<\|F\|^{-1} \leq \bar{\gamma}^{-1}$, we know that $\Pi_{\Omega}(I-\rho F)$ is a contractive operator with the contractive coefficient $1-\bar{\gamma} \rho \in(0,1)$. Banach's mapping principle tell us that $\Pi_{\Omega}(I-\rho F)$ has a unique fixed point, say $x^{*} \in C$, i.e., $x^{*}=\Pi_{\Omega}(I-\rho F)\left(x^{*}\right)$. Since $\left\{w_{n}\right\} \subset C$ is bounded, one supposes that $w_{n_{i}} \rightarrow z \in C$. Since $\lim _{n \rightarrow \infty}\left\|x_{n}-T^{n} y_{n}\right\|=0$ (due to (32)) and $\lim _{n \rightarrow \infty} \alpha_{n}=0$, it follows that

$$
\begin{aligned}
\left\|x_{n}-w_{n}\right\| & \leq\left\|x_{n}-T^{n} y_{n}\right\|+\left\|T^{n} y_{n}-\Pi_{C}\left(I-\alpha_{n} F\right) T^{n} y_{n}\right\| \\
& \leq\left\|x_{n}-T^{n} y_{n}\right\|+\alpha_{n}\left\|F\left(T^{n} y_{n}\right)\right\| \rightarrow 0 \quad(n \rightarrow \infty) .
\end{aligned}
$$

Hence, from $w_{n_{i}} \rightarrow z \in C$ it follows that $x_{n_{i}} \rightarrow z \in C$. Note the situation both $G$ and $\bar{S}$ are nonexpansive operators and that $T$ is an asymptotically nonexpansive operator in the intermediate sense. Since $(I-G) x_{n} \rightarrow 0$ and $(I-\bar{S}) x_{n} \rightarrow 0$ (due to (29) and (37)), by Lemma 11 we have that $z \in \operatorname{Fix}(G)=\operatorname{GSVI}\left(C, B_{1}, B_{2}\right)$ and $z \in \operatorname{Fix}(\bar{S})=\operatorname{Fix}(S)=\bigcap_{n=0}^{\infty} \operatorname{Fix}\left(S_{n}\right)$. From (34), we have that $\lim _{i \rightarrow \infty}\left\|x_{n_{i}}-T x_{n_{i}}\right\|=0$ for the subsequence $\left\{x_{n_{i}}\right\}$ of $\left\{x_{n}\right\}$. It follows from Lemma 6 that $z \in \operatorname{Fix}(T)$. Then, $z \in \Omega=\bigcap_{n=0}^{\infty} \operatorname{Fix}\left(S_{n}\right) \cap \operatorname{GSVI}\left(C, B_{1}, B_{2}\right) \cap \operatorname{Fix}(T)$. Since duality mapping $j(\cdot)$ is weakly sequentially continuous and $w_{n_{i}} \rightarrow z$, we obtain

$$
\limsup _{n \rightarrow \infty}\left\langle F\left(x^{*}\right), j\left(x^{*}-w_{n}\right)\right\rangle=\lim _{i \rightarrow \infty}\left\langle F\left(x^{*}\right), j\left(x^{*}-w_{n_{i}}\right)\right\rangle=\left\langle F\left(x^{*}\right), j\left(x^{*}-z\right)\right\rangle \leq 0,
$$

which implies that (38) holds.

Step 7. One proves that $x_{n} \rightarrow x^{*}$ as $n \rightarrow \infty$. Indeed, putting $p=x^{*}$ in (18) and using (8), we have

$$
\begin{aligned}
\left\|x^{*}-x_{n+1}\right\|^{2} \leq & \beta_{n}\left\|x^{*}-x_{n}\right\|^{2}+\left(1-\beta_{n}\right)\left(1-\alpha_{n} \bar{\gamma}\right)\left\|x^{*}-x_{n}\right\|^{2} \\
& +c_{n}\left(2\left\|x_{n}-x^{*}\right\|+c_{n}\right)+2 \alpha_{n}\left(1-\beta_{n}\right)\left\langle F\left(x^{*}\right), j\left(x^{*}-w_{n}\right)\right\rangle \\
= & {\left[1-\alpha_{n}\left(1-\beta_{n}\right) \bar{\gamma}\right]\left\|x_{n}-x^{*}\right\|^{2}+\alpha_{n}\left(1-\beta_{n}\right) \bar{\gamma} \cdot \frac{2}{\bar{\gamma}}\left\langle F\left(x^{*}\right), j\left(x^{*}-w_{n}\right)\right\rangle } \\
& +c_{n}\left(2\left\|x_{n}-x^{*}\right\|+c_{n}\right) .
\end{aligned}
$$

Since $\sum_{n=0}^{\infty} \alpha_{n}=\infty, \liminf _{n \rightarrow \infty}\left(1-\beta_{n}\right)>0$ (due to conditions (i), (ii)), and $\sum_{n=0}^{\infty} c_{n}<\infty$ (due to the assumption), we deduce from (38) that $\sum_{n=0}^{\infty} \alpha_{n}\left(1-\beta_{n}\right) \bar{\gamma}=\infty, \sum_{n=0}^{\infty} c_{n}\left(2\left\|x_{n}-x^{*}\right\|+c_{n}\right)<\infty$ and $\lim \sup _{n \rightarrow \infty} \frac{2}{\bar{\gamma}}\left\langle F\left(x^{*}\right), j\left(x^{*}-w_{n}\right)\right\rangle \leq 0$. We can employ Lemma 2.12 to the relation (40) and get that $x_{n} \rightarrow x^{*}$ as $n \rightarrow \infty$. This completes the proof.

From [37], we give an example to illustrate the partial condition of Theorem 1 to be satisfied. 
Example 1. Let $H=\mathbf{R}$ with the inner product $\langle a, b\rangle=a b$ and induced norm $\|\cdot\|=|\cdot|$. Let $C=H$, and $T$ be defined as $T x:=\frac{3}{4} \sin x \forall x \in C$. It is not hard to see that for all $x, y \in C$,

$$
\begin{aligned}
\left\|T^{n} x-T^{n} y\right\| & =\frac{3}{4}\left\|\sin T^{n-1} x-\sin T^{n-1} y\right\| \\
& \leq \frac{3}{4}\left\|T^{n-1} x-T^{n-1} y\right\| \\
& \leq \cdots \\
& \leq\left(\frac{3}{4}\right)^{n}\|x-y\| \\
& =\left(1+\theta_{n}\right)\|x-y\|,
\end{aligned}
$$

with $\theta_{n}=\left(\frac{3}{4}\right)^{n}$. Then $T: C \rightarrow C$ is an asymptotically nonexpansive mapping with $\left\{\theta_{n}\right\}$. We take $S_{n}:=T^{n}$ and obtain

$$
\begin{aligned}
\sup _{x \in D}\left\|S_{n} x-S_{n-1} x\right\| & =\sup _{x \in D}\left\|T^{n} x-T^{n-1} x\right\| \\
& \leq\left(\frac{3}{4}\right)^{n-1} \cdot \sup _{x \in D}\|T x-x\| \quad \forall n \geq 1,
\end{aligned}
$$

for any bounded subset $D$ of C. Therefore, it follows that

$$
\sum_{n=1}^{\infty} \sup _{x \in D}\left\|S_{n} x-S_{n-1} x\right\| \leq \sum_{n=1}^{\infty}\left(\frac{3}{4}\right)^{n-1} \cdot \sup _{x \in D}\|T x-x\|<\infty .
$$

In addition, for any sequence $\left\{y_{n}\right\} \subset C$, we get

$$
\begin{aligned}
\left\|T^{n+1} y_{n}-T^{n} y_{n}\right\| & \leq\left(\frac{3}{4}\right)^{n-1}\left\|T^{2} y_{n}-T y_{n}\right\| \\
& =\left(\frac{3}{4}\right)^{n}\left\|\sin T y_{n}-\sin y_{n}\right\| \\
& \leq 2\left(\frac{3}{4}\right)^{n} \rightarrow 0 \quad(n \rightarrow \infty)
\end{aligned}
$$

It is easy to see that Fix $(T)=\{0\}$. We define $S x:=0 \forall x \in C$. It is clear that $S x=\lim _{n \rightarrow \infty} S_{n} x \forall x \in C$ and $\operatorname{Fix}(S)=\bigcap_{n=0}^{\infty} \operatorname{Fix}\left(S_{n}\right)$.

The following results can be easily obtained by virtue of the argument techniques in Theorem 1 , and hence we omit the details.

Theorem 2. Fix a bounded, convex, nonempty, and closed set in a 2-uniformly smooth and uniformly convex Banach space E. We suppose $E$ has a weakly sequentially continuous duality mapping. Let $\Pi_{C}: E \rightarrow C$ be a nonexpansive sunny retraction. Let the accretive mappings $B_{1}, B_{2}: C \rightarrow E$ be $\alpha$-inverse-strongly and $\beta$-inverse-strongly. Let linear bounded operator $F$ be a strongly positive on $E$ with coefficient $\bar{\gamma}>0$. Let $T$ be an asymptotically nonexpansive self-mapping on $C$ with a sequence $\left\{\theta_{n}\right\} \subset[0, \infty)$ satisfying $\sum_{n=0}^{\infty} \theta_{n}<\infty$, and $\left\{S_{n}\right\}_{n=0}^{\infty}: C \rightarrow C$ be a countable family of $\ell$-uniformly Lipschitzian pseudocontractive mappings such that $\Omega:=\bigcap_{n=0}^{\infty} \operatorname{Fix}\left(S_{n}\right) \cap \operatorname{GSVI}\left(C, B_{1}, B_{2}\right) \cap \operatorname{Fix}(T) \neq \varnothing$ where $\operatorname{GSVI}\left(C, B_{1}, B_{2}\right)$ is the fixed-point set of the mapping $G:=\Pi_{C}\left(I-\mu_{1} B_{1}\right) \Pi_{C}\left(I-\mu_{2} B_{2}\right)$ with $0<\mu_{1}<\frac{\alpha}{\kappa^{2}}$ and $0<\mu_{2}<\frac{\beta}{\kappa^{2}}$. For arbitrarily given $x_{0} \in C$, let $\left\{x_{n}\right\}$ be the sequence given by

$$
\left\{\begin{array}{l}
u_{n}=\gamma_{n}\left(x_{n}-S_{n} u_{n}\right)+S_{n} u_{n} \\
z_{n}=\Pi_{C}\left(u_{n}-\mu_{2} B_{2} u_{n}\right) \\
y_{n}=\Pi_{C}\left(z_{n}-\mu_{1} B_{1} z_{n}\right) \\
x_{n+1}=\left(1-\beta_{n}\right) \Pi_{C}\left(T^{n} y_{n}-\alpha_{n} F T^{n} y_{n}\right)+\beta_{n} x_{n}, \quad \forall n \geq 0
\end{array}\right.
$$


where $\left\{\alpha_{n}\right\},\left\{\beta_{n}\right\}$ and $\left\{\gamma_{n}\right\}$ are the sequences in $[0,1]$ satisfying the following conditions:

(i) $\lim _{n \rightarrow \infty} \alpha_{n}=0$ and $\sum_{n=0}^{\infty} \alpha_{n}=\infty$;

(ii) $1 \geq \lim \sup _{n \rightarrow \infty} \beta_{n} \geq \liminf _{n \rightarrow \infty} \beta_{n} \geq 0$;

(iii) $1 \geq \limsup _{n \rightarrow \infty} \gamma_{n} \geq \liminf _{n \rightarrow \infty} \gamma_{n} \geq 0$ and $\lim _{n \rightarrow \infty}\left|\gamma_{n+1}-\gamma_{n}\right|=0$.

Assume that $\sum_{n=0}^{\infty} \sup _{x \in C}\left\|S_{n+1} x-S_{n} x\right\|<\infty$, and let $S$ be a mapping of $C$ into itself defined by $S x=\lim _{n \rightarrow \infty} S_{n} x$ for all $x \in C$, and suppose that $\operatorname{Fix}(S)=\bigcap_{n=0}^{\infty} \operatorname{Fix}\left(S_{n}\right)$. Then $\left\{x_{n}\right\}$ converges strongly to $x^{*} \in \Omega$ provided $T^{n+1} y_{n}-T^{n} y_{n} \rightarrow 0$ as $n \rightarrow \infty$. In this case,

(a) $x^{*} \in \Omega$ solves the VI: $\left\langle F\left(x^{*}\right), j\left(x^{*}-x\right)\right\rangle \leq 0, \forall x \in \Omega$;

(b) $\left(x^{*}, y^{*}\right)$ solves the GSVI (3) with $y^{*}=\Pi_{C}\left(I-\mu_{2} B_{2}\right) x^{*}$.

Proof. Since the set $C$ is bounded, one gets $\operatorname{diam}(C)=\sup _{x, y \in C}\|x-y\|<\infty$. Please note that $T$ is an asymptotically nonexpansive mapping equipped $\left\{\theta_{n}\right\} \subset[0, \infty)$ satisfying $\sum_{n=0}^{\infty} \theta_{n}<\infty$. Then, we deduce that for all $x, y \in C$,

$$
\left\|T^{n} x-T^{n} y\right\| \leq\left(1+\theta_{n}\right)\|x-y\|=\|x-y\|+\theta_{n}\|x-y\| \leq\|x-y\|+\theta_{n} \operatorname{diam}(C) .
$$

Hence, we get $c_{n}:=\max \left\{0, \sup _{x, y \in C}\left(\left\|T^{n} x-T^{n} y\right\|-\|x-y\|\right)\right\} \leq \theta_{n} \operatorname{diam}(C)$, which immediately attains $\sum_{n=0}^{\infty} c_{n}<\infty$. Therefore, by Theorem 1 we derive the result.

Theorem 3. Fix a convex, nonempty closed set in a real Hilbert space H. Let the monotone mappings $B_{1}, B_{2}: C \rightarrow H$ be $\alpha$-inverse-strongly and $\beta$-inverse-strongly. Set $F: C \rightarrow H$ a $\kappa$-Lipschitzian and $\eta$-strongly monotone with constants $\kappa, \eta>0$ such that $\tau=1-\sqrt{1-\rho\left(2 \eta-\rho \kappa^{2}\right)} \in(0,1]$, where $0<$ $\rho<\frac{2 \eta}{\kappa^{2}}$. Let $T$ be an asymptotically nonexpansive mapping equipped with an asymptotic sequence $\left\{\theta_{n}\right\}$. Let $\left\{S_{n}\right\}_{n=0}^{\infty}: C \rightarrow C$ be a countable family of $\ell$-uniformly Lipschitzian pseudocontractions such that $\Omega:=$ $\bigcap_{n=0}^{\infty} \operatorname{Fix}\left(S_{n}\right) \cap \operatorname{GSVI}\left(C, B_{1}, B_{2}\right) \cap \operatorname{Fix}(T) \neq \varnothing$ where $\operatorname{GSVI}\left(C, B_{1}, B_{2}\right)$ is the fixed-point set of the mapping $G:=P_{C}\left(I-\mu_{1} B_{1}\right) P_{C}\left(I-\mu_{2} B_{2}\right)$ with $\mu_{1} \in(0,2 \alpha)$ and $\mu_{2} \in(0,2 \beta)$. For any given $x_{0} \in C$, let $\left\{x_{n}\right\}$ be the sequence given by

$$
\left\{\begin{array}{l}
u_{n}=\gamma_{n}\left(x_{n}-S_{n} u_{n}\right)+S_{n} u_{n}, \\
z_{n}=P_{C}\left(u_{n}-\mu_{2} B_{2} u_{n}\right) \\
y_{n}=P_{C}\left(z_{n}-\mu_{1} B_{1} z_{n}\right) \\
x_{n+1}=\left(1-\beta_{n}\right) P_{C}\left(T^{n} y_{n}-\alpha_{n} \rho F T^{n} y_{n}\right)+\beta_{n} x_{n}, \quad \forall n \geq 0
\end{array}\right.
$$

where $\left\{\alpha_{n}\right\},\left\{\beta_{n}\right\}$ and $\left\{\gamma_{n}\right\}$ are the sequences in $(0,1]$ satisfying the following conditions:

(i) $\lim _{n \rightarrow \infty} \alpha_{n}=0$ and $\sum_{n=0}^{\infty} \alpha_{n}=\infty$;

(ii) $\lim _{n \rightarrow \infty} \frac{\theta_{n}}{\alpha_{n}}=0$;

(iii) $1 \geq \lim \sup _{n \rightarrow \infty} \beta_{n} \geq \liminf _{n \rightarrow \infty} \beta_{n} \geq 0$;

(iv) $1 \geq \lim \sup _{n \rightarrow \infty} \gamma_{n} \geq \liminf _{n \rightarrow \infty} \gamma_{n} \geq 0$ and $\lim _{n \rightarrow \infty}\left|\gamma_{n+1}-\gamma_{n}\right|=0$.

Assume that $\sum_{n=1}^{\infty} \sup _{x \in D}\left\|S_{n} x-S_{n-1} x\right\|<\infty$ for any bounded subset $D$ of $C$, and let $S: C \rightarrow C$ be a mapping defined by $S x=\lim _{n \rightarrow \infty} S_{n} x$ for all $x \in C$, and suppose that $\operatorname{Fix}(S)=\bigcap_{n=0}^{\infty} \operatorname{Fix}\left(S_{n}\right)$. Then $\left\{x_{n}\right\}$ converges strongly to $x^{*} \in \Omega$ provided $\lim _{n \rightarrow \infty}\left\|T^{n+1} y_{n}-T^{n} y_{n}\right\|=0$. In this case,

(a) $x^{*} \in \Omega$ solves the VI: $\left\langle F\left(x^{*}\right), x^{*}-x\right\rangle \leq 0, \forall x \in \Omega$;

(b) $\left(x^{*}, y^{*}\right)$ solves the GSVI (3) with $y^{*}=P_{C}\left(x^{*}-\mu_{2} B_{2} x^{*}\right)$.

\section{Conclusions}

In this paper, we studied a relaxed Mann implicit iteration method for the solution of a GSVI. It deserves mentioning that our iteration involves an infinite family of operators and there is no 
commutative condition to be required. With the aid of the projection, we established a convergence result in setting of 2-uniformly smooth and uniformly convex spaces.

Author Contributions: These authors contributed equally to this work.

Funding: This paper was supported by the National Natural Science Foundation of China under Grant 11601348.

Conflicts of Interest: The authors declare no conflict of interest.

\section{References}

1. Goebel, K.; Kirk, W.A. A fixed point theorem for asymptotically nonexpansive mappings. Proc. Am. Math. Soc. 1972, 35, 171-174. [CrossRef]

2. Kaczor, W.; Kuczumow, T.; Reich, S. A mean ergodic theorem for mappings which are asymptotically nonexpansive in the intermediate sense. Nonlinear Anal. 2001, 47, 2731-2742. [CrossRef]

3. Takahashi, W.; Wen, C.F.; Yao, J.C. The shrinking projection method for a finite family of demimetric mappings with variational inequality problems in a Hilbert space. Fixed Point Theory 2018, 19, 407-419. [CrossRef]

4. Qin, X.; Cho, S.Y.; Wang, L. Iterative algorithms with errors for zero points of m-accretive operators. Fixed Point Theory Appl. 2013, 2013, 148. [CrossRef]

5. Ceng, L.C.; Lur, Y.Y.; Wen, C.F. Implicit and explicit iterative algorithms for hierarchical variational inequality in uniformly smooth Banach spaces. J. Inequal. Appl. 2017, 2017, 247. [CrossRef] [PubMed]

6. Wang, Q.W.; Guan, J.L.; Ceng, L.C.; Hu, B. General iterative methods for systems of variational inequalities with the constraints of generalized mixed equilibria and fixed point problem of pseudocontractions. J. Inequal. Appl. 2018, 2018, 315. [CrossRef]

7. Ceng, L.C.; Gupta, H.; Ansari, Q.H. Implicit and explicit algorithms for a system of nonlinear variational inequalities in Banach spaces. J. Nonlinear Convex Anal. 2015, 16, 965-984.

8. Zhao, X.; Ng, K.F.; Li, C.; Yao, J.C. Linear regularity and linear convergence of projection-based methods for solving convex feasibility problems. Appl. Math. Optim. 2018, 78, 613-641. [CrossRef]

9. Ansari, Q.H.; Babu, F.; Yao, J.C. Regularization of proximal point algorithms in Hadamard manifolds. J. Fixed Point Theory Appl. 2019, 21, 25. [CrossRef]

10. Guan, J.L.; Ceng, L.C.; Hu, B. Strong convergence theorem for split monotone variational inclusion with constraints of variational inequalities and fixed point problems. J. Inequal. Appl. 2018, 2018, 311. [CrossRef]

11. Nguyen, L.V.; Qin, X. Some results on strongly pseudomonotone quasi-variational inequalities. Set-Valued Var. Anal. 2019. [CrossRef]

12. Al-Mezel, S.A.; Ansari, Q.H.; Ceng, L.C. Hybrid viscosity approach for hierarchical fixed-point problems. Appl. Anal. 2015, 94, 2-23. [CrossRef]

13. Qin, X.; Petrusel, A.; Yao, J.C. CQ iterative algorithms for fixed points of nonexpansive mappings and split feasibility problems in Hilbert spaces. J. Nonlinear Convex Anal. 2018, 19, 157-165.

14. Qin, X.; Cho, S.Y. Convergence analysis of a monotone projection algorithm in reflexive Banach spaces. Acta Math. Sci. 2017, 37, 488-502. [CrossRef]

15. Kato, T. Nonlinear semigroups and evolution equations. J. Math. Soc. Jpn. 1967, 19, 508-520. [CrossRef]

16. Ceng, L.C. Convergence analysis of a Mann-like iterative algorithm in reflexive Banach spaces. Appl. Set-Valued Anal. Optim. 2019, 1, 1-18.

17. Qin, X.; Cho, S.Y.; Wang, L. Strong convergence of an iterative algorithm involving nonlinear mappings of nonexpansive and accretive type. Optimization 2018, 67, 1377-1388. [CrossRef]

18. Dehaish, B.A.B. Weak and strong convergence of algorithms for the sum of two accretive operators with applications. J. Nonlinear Convex Anal. 2015, 16, 1321-1336.

19. Qin, X.; Yao, J.C. Projection splitting algorithms for nonself operators. J. Nonlinear Convex Anal. 2017, 18, 925-935.

20. Chang, S.S.; Wen, C.F.; Yao, J.C. Common zero point for a finite family of inclusion problems of accretive mappings in Banach spaces. Optimization 2018, 67, 1183-1196. [CrossRef]

21. Ceng, L.C.; Petrusel, A.; Yao, J.C.; Yao, Y. Hybrid viscosity extragradient method for systems of variational inequalities, fixed points of nonexpansive mappings, zero points of accretive operators in Banach spaces. Fixed Point Theory 2018, 19, 487-501. [CrossRef] 
22. Qin, X.; Yao, J.C. Weak convergence of a Mann-like algorithm for nonexpansive and accretive operators. J. Inequal. Appl. 2016, 2016, 232. [CrossRef]

23. Ceng, L.C.; Wang, C.Y.; Yao, J.C. Strong convergence theorems by a relaxed extragradient method for a general system of variational inequalities. Math. Methods Oper. Res. 2008, 67, 375-390. [CrossRef]

24. Cai, G.; Shehu, Y.; Iyiola, O.S. Samuel Viscosity iterative algorithms for fixed point problems of asymptotically nonexpansive mappings in the intermediate sense and variational inequality problems in Banach spaces. Numer. Algorithms 2017, 76, 521-553. [CrossRef]

25. Ceng, L.C.; Petrusel, A.; Yao, J.C.; Yao, Y. Systems of variational inequalities with hierarchical variational equality constraints for Lipschitzian pseudocontractions. Fixed Point Theory 2019, 20, 113-133. [CrossRef]

26. Liu, L. Iterative methods for fixed points and zero points of nonlinear mappings with applications. Optimization 2019. [CrossRef]

27. Reich, R. Weak convergence theorems for nonexpansive mappings in Banach spaces. J. Math. Anal. Appl. 1979, 67, 274-276. [CrossRef]

28. Deimling, K. Zeros of accretive operators. Manuscr. Math. 1974, 13, 365-374. [CrossRef]

29. Gossez, J.P.; Dozo, E.L. Some geometric properties related to the fixed point theory for nonexpansive mappings. Pac. J. Math. 1972, 40, 565-573. [CrossRef]

30. Xu, H.K. Inequalities in Banach spaces with applications. Nonlinear Anal. 1991, 16, 1127-1138. [CrossRef]

31. Aoyama, K.; Kimura, Y.; Takahashi, W.; Toyoda, M. Approximation of common fixed points of a countable family of nonexpansive mappings in a Banach space. Nonlinear Anal. 2007, 67, 2350-2360 [CrossRef]

32. Oka, H. An ergodic theorem for asymptotically nonexpansive mappings in the intermediate sense. Proc. Am. Math. Soc. 1997, 125, 1693-1703. [CrossRef]

33. Suzuki, T. Strong convergence of Krasnoselskii and Mann's type sequences for one-parameter nonexpansive semigroups without Bochner integrals. J. Math. Anal. Appl. 2005, 305, 227-239. [CrossRef]

34. Marino, G.; Xu, H.K. A general iterative method for nonexpansive mappings in Hilbert spaces. J. Math. Anal. Appl. 2006, 318, 43-52. [CrossRef]

35. Goebel, K.; Kirk, W.A. Topics in Metric Fixed Point Theory; Cambridge Studies in Advanced Mathematics; Cambridge University Press: Cambridge, UK, 1990; Volume 28.

36. Xue, Z.; Zhou, H.; Cho, Y.J. Iterative solutions of nonlinear equations for $\mathrm{m}$-accretive operators in Banach spaces. J. Nonlinear Convex Anal. 2000, 1, 313-320.

37. Ceng, L.C.; Wen, C.F. Systems of variational inequalities with hierarchical variational inequality conxtraints for asymptotically nonexpansive and pseudocontractive mappings. RACSAM 2019. [CrossRef]

(C) 2019 by the authors. Licensee MDPI, Basel, Switzerland. This article is an open access article distributed under the terms and conditions of the Creative Commons Attribution (CC BY) license (http://creativecommons.org/licenses/by/4.0/). 